\title{
Arthur Groos / Hans-Jochen Schiewer (Hgg.), Kulturen des Manuskriptzeitalters. 2004
}

\author{
Herberichs, Cornelia
}

\begin{abstract}
Der Sammelband vereint Beiträge einer Arbeitstagung nordamerikanischer und deutscher Mediävistinnen und Mediävisten. Die umfangreiche Publikation dokumentiert jedoch nicht nur diese internationale Konferenz; indem sie eine Reihe eröffnet, manifestiert sie zugleich den Beginn eines auf Dauer und Kontinuität gestellten transkontinentalen Dialoges. Die Themenwahl für die Arbeitstagung deutet auf eine programmatische Offenheit, was das thematische, zeitliche wie methodische Spektrum betrifft. Dementsprechend heterogen gestaltet sich der Band, der jeweils sieben deutsch- und englischsprachige Beiträge enthält
\end{abstract}

DOI: https://doi.org/10.1515/arbi.2006.158

Posted at the Zurich Open Repository and Archive, University of Zurich ZORA URL: https://doi.org/10.5167/uzh-154570

Journal Article

Published Version

Originally published at:

Herberichs, Cornelia (2006). Arthur Groos / Hans-Jochen Schiewer (Hgg.), Kulturen des Manuskriptzeitalters. 2004. Arbitrium: Zeitschrift für Rezensionen zur germanistischen Literaturwissenschaft, 24(2):158161.

DOI: https://doi.org/10.1515/arbi.2006.158 
Arthur Groos / Hans-Jochen Schiewer (Hgg.), Kulturen des Manuskriptzeitalters. Ergebnisse der Amerikanisch-Deutschen Arbeitstagung an der Georg-August-Universität Göttingen vom 17. bis 20. Oktober 2002. (Transatlantische Studien zu Mittelalter und Früher Neuzeit I) Vandenhoeck \& Ruprecht, Göttingen 2004. 364 S., € 39,90.

Der Sammelband vereint Beiträge einer Arbeitstagung nordamerikanischer und deutscher Mediävistinnen und Mediävisten. Die umfangreiche Publikation dokumentiert jedoch nicht nur diese internationale Konferenz; indem sie eine Reihe eröffnet, manifestiert sie zugleich den Beginn eines auf Dauer und Kontinuität gestellten transkontinentalen Dialoges. Die Themenwahl für die Arbeitstagung deutet auf eine programmatische Offenheit, was das thematische, zeitliche wie methodische Spektrum betrifft. Dementsprechend heterogen gestaltet sich der Band, der jeweils sieben deutsch- und englischsprachige Beiträge enthält.

Dem Phänomen der Textvariabilität geht die Studie zur handschriftlichen Überlieferung des Parzival von Martin Baisch nach („Die Bedeutung der Varianz. Zu den auktorialen Selbstentwür- 
fen im Parzival Wolframs von Eschenbach“, S. I I - 39). Baisch untersucht die divergenten Überlieferungen und eröffnet derart eine zweifache Perspektive auf mittelalterliche Autorschaft: Werfen Varianten methodisch die Frage nach dem historischen Status von Autorschaft auf, so thematisieren insbesondere die von Baisch fokussierten metapoetischen Passagen des Parzival romanintern das Thema Autorschaft. Textvarianz insbesondere in diesen Passagen verdiene daher besondere Aufmerksamkeit, ermögliche sie doch „eine Wahrnehmung, welche die Überlieferung bei der Problembewältigung beobachtet“ (S. 26). Einsichtig wird derart, daß Varianz „auf die Schwierigkeiten einer sich Freiräume schaffenden volkssprachlichen Literatur“ (S. 39) hinweist, wo diese in literaturtheoretischer Reflexion eigene Wege beschreitet.

Kirsten M. Christensen macht unter dem Titel „Poetic Piety. The Interplay of Mysticism and Catechism in the Late Middle Ages" (S. 4I-59) auf Exempel gegenseitiger Durchdringung zweier Texttypen mit unterschiedlicher pragmatischer Ausrichtung aufmerksam: Katechetische Texte, womit hier allerdings vornehmlich das Credo und Paternoster gemeint sind, und solche mystischer Prägung wiesen gegenseitige Anleihen an sprachlichen Formeln und Textstrukturen auf. Dies sei dem Bestreben geschuldet „a fusion of institutional legitimacy“ (S. 57) zu erreichen.

Arthur Groos initiiert in seinem Aufsatz „Orientalizing the Medieval Orient. The East in Wolfram von Eschenbach's Parzival“ (S. 6I -86) eine kritische Debatte mit dem kulturwissenschaftlichen Konzept des „orientalism“, indem er aus mediävistischer Perspektive dessen teleologische Prämissen hinsichtlich der Vormoderne aufdeckt. Die schematischen Thesen Edward Saids zur Vormoderne konfrontiert Groos mit den komplexen Entwürfen interkultureller Begegnung in der Literatur, in der sich "medieval counter-narratives to the dominant clerical crusading discourse“ (S. 65) ausformulierten. Eine subtile Lektüre der Gahmuret-Bücher arbeitet die Formbarkeit des Diskurses im Mittelalter heraus, welche den hohen Komplexitätsgrad der Reflexion belegt.

Ein Beitrag von Albrecht Hausmann zum „Bild zu Süßkind von Trimberg in der Manessischen Liederhandschrift" (S. 87- I I2) liefert eine präzise Bildlektüre der vielschichtigen Autorminiatur. Hausmann fördert die Interdependenz makrostruktureller und mikrostruktureller Bildlogiken an den Tag. Vor der Folie der „,Bildwelt‘ der Manessischen Liederhandschrift“ (S. 94) entziffert Hausmann ikonographische Identifikationssignale der Figuren und deutet die kalkulierte Störung des symmetrischen Bildaufbaus: Mit diesen visuellen Elementen habe der Maler eine Dynamisierung der theologischen Disputation zwischen Vertretern der Christenheit und der jüdischen Figur ins Bild gesetzt. Die kulturgeschichtliche Perspektive, die sich mittels einer solchen Bildinterpretation öffnet, trägt in das Stereotyp vom aggressiv-antagonistischen Verhältnis von Juden und Christen im Mittelalter eine kleine, doch sehr bedeutsame Differenzierung ein.

Gert Hübners „Überlegungen zur Historizität von Metapherntheorien“ (S. I I3-I 53) stellen methodische Prämissen der literaturwissenschaftlichen Metapherninterpretation zur Diskussion. Nach der Interdependenz von zeitgenössischer Metapherntheorie und poetischem Metapherngebrauch fragend, vertritt Hübner dezidiert die Forderung nach einer angemessenen Berücksichtigung des herrschenden „erkenntnistheoretischen Paradigma[s]“ (S. I53) der Zeit. Eine Metapher sei im Mittelalter demnach nicht eine „Figur der Einbildungskraft“, wie dies die Ästhetiktheorie des I 8. Jahrhunderts reflektiert habe (S. I 25), und ihre Mehrdeutigkeit kein Indiz für Bedeutungsoffenheit, wie dies die Interaktionstheorie der Moderne fasse. Der Metapherngebrauch mittelalterlicher, volkssprachlicher Autoren sei vielmehr an die Substitutionsthese zurückzubinden, die sich in lateinischen theologischen und rhetorischen Schriften finde. Es erscheint allerdings erwägenswert, ob Metaphorologie den Interpretationsrahmen poetischer Metaphern vollständig zu definieren vermag oder ob von Ungleichzeitigkeiten und literarischen Freiräumen ausgegangen werden kann und soll.

Dem Profil der Germanistik im amerikanischen Ausland entsprechend ist in dem Sammelband auch ein nordistischer Beitrag vertreten: Marianne Kalinke beschäftigt sich mit einer mutmaßlich „Lost German Literature in Icelandic Translation. The Legends of King Oswald and Emperor Henry II" (S. I55- I80). Eine Reihe von Indizien - die Bebilderung im frühneuzeitlichen Druck und historiographische Befunde - sprechen dafür, daß die erhaltenen deutschsprachigen Versionen der Oswald-Legenden nur bruchstückhaft den Gesamtbestand mittelalterlicher Versionen repräsentieren.

Illustrationen epischer Stoffe spielen auch in William Layhers Beitrag eine Rolle: „Siegfried the Giant. Heroic Representation and the Amplified Body in the Heldenbuch" (S. I8I-213). Layher weist darauf hin, daß dem Körper des Helden im späten Mittelalter in mehrfachem Sinne 
besondere Evidenz zuteil werde. Da sich die Wörter rise und helt in ihren Bedeutungen einander angenähert haben, wird die Konzeptualisierung der Physis des Helden als gigantischer gefördert. Es kommt zu Diskrepanzen zwischen Visualisierungen und Narration, aber auch zu sekundären Umschriften der Deskriptio. Das Bedürfnis nach empirischer Evidenz der Reliquien schließlich zeige den veränderten Umgang mit den heldenepischen Stoffen an.

Eine kritische Bewertung des Begriffs ,New Philology' unternimmt Freimut Löser in seinem Beitrag („Postmodernes Mittelalter? ,New Philology“ und ,Überlieferungsgeschichte“", S. 2 I 5236). Die Forderung Cerquiglinis, historisch adäquate Texteditionen mit Hilfe neuester Technologien herzustellen, unterzieht Löser einer dezidierten Kritik (S. 220). Er skizziert den relevanten literaturwissenschaftlichen Diskurs und verweist auf Publikationen, die eine unbekannte Vorgeschichte der ,New Philology' vor Augen treten lassen. Vor diesem Hintergrund erscheine das Postulat der programmatischen Neuheit der ,New Philology' fragwürdig, ohne daß Löser die Gültigkeit und Notwendigkeit des methodischen Ansatzes bestreitet, indessen seine Basis sogar erweitert (S. 236). Angesichts der prekären Dynamik wissenschaftlicher Gruppenbildung stellen Lösers Darlegungen ein konstruktives und ermutigendes Plädoyer dafür dar, existierende Brücken zu entdecken, die über terminologische Gräben hinwegführen.

Volker Mertens erhellt in seinem Beitrag zu "Geschichte und Geschichten um den Gral“ (S. 237-258) die Funktion der realhistorischen Anspielungen in Wolframs Parzival. Mit der Verwendung außerliterarischer Namen und Motive nutze Wolfram den verificatio-Topos (S. 237) und lasse diesen doch in mehrfacher Überschreitung hinter sich (S. 239f.). Mertens ortet unter dieser Fragestellung eine Reihe von Indizien, die eine Spätdatierung des Parzival bedenkenswert erscheinen lassen. Auch führen die Überlegungen zum Status des Geschichtlichen zu einer Neubewertung von Wolframs Gral-Konzeption, die als subtile Antwort auf konkurrierende literarische Geschichtskonzepte der französischsprachigen Stoffversionen erscheint: Zwischen der „mythischen Unbestimmtheit Chrétiens" und der "heilswahre[n]" Historisierung " des Prosa-Perceval (S. 255 ) sei demnach die spezifische Position Wolframs zu verorten. Unabhängig von einer möglichen, empirisch jedoch kaum zu belegenden Intertextualität erhellt Mertens' Konstruktion dieses literarischen Dreiecks Wolframs spezifische Gralsdarstellung, profiliert Wolframs Antwort auf eine inhärente Aporie.

Matthias Meyer verfolgt eine methodische Fragestellung an einem konkreten Beispiel, indem er Spuren der Instanz mittelalterlicher Literaturproduktion in seinen literarischen Repräsentationen verfolgt („Speculum narrationis. Erzählte Sexualität im Spiegel von Der Spiegel und Spiegel und Igel“, S. 259-277): Im Spannungsfeld von Mündlichkeit und Schriftlichkeit seien ,Schreiber die „Protagonisten dieser Bimedialität“ (S. 260). Die Präsenz des schreiber im Epimythion des Spiegel und implizite autoreferentielle Passagen des Märe erlaubten es, das Moment der Narrativierung von Ereignissen als einen Gegenstand der Textpoetik zu betrachten. In seiner markierten Entzogenheit stelle der Schreiber die „,dekonstruierte Hauptfigur““ (S. 262) dar, aus der die Selbstlegitimation der Geschichte wie die Ambiguisierung ihrer Didaxe resultiere. Augenfällig mache den Konnex von Handlung und Verschriftlichung insbesondere der Vergleich mit dem Märe Spiegel und Igel, dessen Aussparung von Reflexen auf die Autorinstanz mit der Vereindeutigung der Moral einhergehe. Meyers Beitrag macht die notwendige Verschränkung historischer Kontextualisierung mit textimmanenter Interpretation augenfällig, wenn adäquate Aussagen über die literarische Selbstreferenzialität getroffen werden sollen.

Marian Polhill beschäftigt sich in ihrem Beitrag "Scientific' Bestiaries. Translations, Misreadings, and Cultural Significance“ (S. 279-297) mit den Adaptationsprozessen volkssprachiger Naturkunde. Philologisch präzise Vergleiche des Buchs der Natur Konrads von Megenberg und des Tierbuchs Hans Minners mit deren Vorlagen geben Aufschluß über kulturelle Rahmensituationen der übersetzten und bearbeiteten Werke: Antijüdische Invektiven, aber auch Legitimationsstrategien medizinischer Berufsgruppen fordern dazu heraus "to re-examine how the medieval texts themselves interconnect with broader cultural dicourses" (S. 280). Polhills Ankündigung zukünftiger, ausführlicherer Studien auf diesem Gebiet läßt eine Intensivierung von Interdisziplinarität in diesem Feld der Wissensgeschichte erwarten.

James Rushing verortet das Phänomen der Handschriftenillustration an der Schnittfläche zwischen illiterater und litterater Literaturrezeption anhand der Aeneis-Illustrationen der Antike, des lateinischen und des volkssprachigen Mittelalters („More Images at the Interface. Aeneas in the Visual Arts“, S. 299-320). Rushing geht dabei von einer "division of medieval world into two 
cultural spheres or epistemes" (S. 299) aus, eine methodisch wenig präzise Prämisse, die gewisse Zirkelschlüsse nicht vermeiden kann: Bilder als Verständnishilfen für Texte zu deklarieren und demnach vom Text-Bild-Verhältnis illustrierter Handschriften auf die Lesefähigkeit der Leserschaft zu schließen (vgl. S. 317-319) verengt nicht nur den Blick auf die Funktion von Buchillustrationen, sondern personalisiert auch zu stark die Semioralität des Mittelalters („,[weak] readers who may need some help from pictures“, S. 3 I 8f.). Die Einschränkungen, die Rushing am Ende seines Artikels in Bezug auf seine „interface theory“ einräumt, stehen unverbunden den affirmativen Ausführungen des Beitrags gegenüber.

Uta Störmer-Caysa stellt in ihrer Studie zur Erzähltechnik der Crone deren Zeitgestaltung in den Mittelpunkt: Den Phänomenen inkohärenter Handlungssukzessionen wird auf der Handlungs- und Figurenebene nachgegangen, räumliche Strukturen und Figurenkonstellationen werden auf die Zeitdarstellung bezogen. Es eröffnen sich interessante und anregende Einsichten in die Mehrschichtigkeit des Dargestellten, das Nebeneinander von linearer und nichtlinearer Darstellung und die literarische Kombination konkurrierender Deutungsmuster (S. $338 f$.). Dieser Poetik liege die „Denkfigur einer Rezeption“ zugrunde, die „Alternativen mit- und nachvollzieht“, nämlich die komplexe Denkfigur der „Konditionalität“ (S. $339 f$.), die das Text- und Zeitverständnis weder auf die mythische noch auf die rationale Logik festlege.

Sarah Westphal geht im den Sammelband abschließenden Beitrag „Virtues in Print“ (S. 34I 364) der Textgeschichte Hermanns von Sachsenheim Mörin nach. Westphal korreliert die soziokulturellen Kontexte der Druckfassung mit der Liebeskonzeption, die der früheste Druck transportiert: Die gesellschaftlichen Prozesse in den ersten Jahrzehnten des I6. Jahrhunderts determinierten demnach die Textallianz, in welche die Minnerede im Druck von I 5 I 2 eingepaßt wird, in der eine misogyne Ekloge mit einem Lob auf den Ehestand ersetzt wird.

Daß die neu gegründete Reihe „Transatlantische Studien zu Mittelalter und Früher Neuzeit“ auf einen Mangel im germanistischen Forschungsdiskurs reagiert, ist angesichts der weit verbreiteten Abschottung der nationalen Germanistiken offensichtlich. Auch wenn die Literaturhinweise in den Fußnoten die internationale, vielfältige Länder- und Sprachgrenzen überschreitende Auseinandersetzung, die dem Band bereits vorausliegt, bezeugen, zeigen sie mitunter auch, daß dies noch zu punktuell geschieht. Bekanntlich zeitigen persönlicher Austausch und gemeinsame Anwesenheit bei einer Tagung überdies Kommunikationseffekte, die sich sonst nur schwächer, vermittelter und langwieriger gestalten; die punktuellen Vernetzungen der Artikel untereinander belegen diese Produktivität des wissenschaftlichen Gesprächs. Um diesen Austausch allerdings zu intensivieren, wäre eine engere Definition des im Arbeitsgespräch fokussierten Gegenstandes oder Themas förderlich.

Universität Zürich

Cornelia Herberichs

Deutsches Seminar

Schönberggasse 9

$\mathrm{CH}-800$ I Zürich

cornelia.herberichs@access.unizh.ch 